\title{
A!
}

This is an electronic reprint of the original article.

This reprint may differ from the original in pagination and typographic detail.

Solinas, Paolo; Averin, Dmitri V.; Pekola, Jukka P.

\section{Work and its fluctuations in a driven quantum system}

Published in:

Physical Review B

DOI:

10.1103/PhysRevB.87.060508

Published: 25/02/2013

Document Version

Publisher's PDF, also known as Version of record

Please cite the original version:

Solinas, P., Averin, D. V., \& Pekola, J. P. (2013). Work and its fluctuations in a driven quantum system. Physical Review B, 87(6), 1-5. [060508]. https://doi.org/10.1103/PhysRevB.87.060508

This material is protected by copyright and other intellectual property rights, and duplication or sale of all or part of any of the repository collections is not permitted, except that material may be duplicated by you for your research use or educational purposes in electronic or print form. You must obtain permission for any other use. Electronic or print copies may not be offered, whether for sale or otherwise to anyone who is not an authorised user. 


\title{
Work and its fluctuations in a driven quantum system
}

\author{
Paolo Solinas, ${ }^{1,2}$ Dmitri V. Averin, ${ }^{3}$ and Jukka P. Pekola ${ }^{1}$ \\ ${ }^{1}$ Low Temperature Laboratory (OVLL), Aalto University School of Science, P.O. Box 13500, 00076 Aalto, Finland \\ ${ }^{2}$ Department of Applied Physics, Aalto University School of Science, P.O. Box 11000, 00076 Aalto, Finland \\ ${ }^{3}$ Department of Physics and Astronomy, Stony Brook University, SUNY, Stony Brook, New York 11794-3800, USA
}

(Received 22 June 2012; revised manuscript received 18 December 2012; published 25 February 2013)

\begin{abstract}
We analyze work done on a quantum system driven by a control field. The average work depends on the whole dynamics of the system, and is obtained as the integral of the average power operator. As a specific example we focus on a superconducting Cooper-pair box forming a two-level system. We obtain expressions for the average work and work distribution in a closed system, and discuss control field and environment contributions to the average work for an open system.
\end{abstract}

DOI: 10.1103/PhysRevB.87.060508

PACS number(s): 74.78.Na, 05.40.-a

The fluctuation relations (FRs) ${ }^{1,2}$ govern work and dissipation in small classical systems when they are driven out of equilibrium. They have recently attracted much attention because of their applications in molecular systems. ${ }^{3}$ Fluctuation relations can also be accurately studied in single-electron transport. ${ }^{4-6} \mathrm{~A}$ natural question is if similar concepts and experiments can be extended to the quantum regime. The first attempts in this direction focused on finding a proper work operator. ${ }^{1,7-10}$ However, after a long debate, it has become clear that this approach has serious drawbacks. ${ }^{11}$ Work is characterized by a process, not only by the instantaneous state of the system, ${ }^{11,12}$ and therefore it cannot be defined by an operator local in time, which would disregard the actual evolution of the system under the driving protocol. Although this is not an issue for closed systems it can become critical when discussing work in open systems. Alternatively the work has been defined through a two-measurement process (TMP). ${ }^{10-16}$ The energy of the system is measured at the beginning and at the end of the evolution and the work done in a process is determined by the corresponding energy difference. This definition has the advantage that the quantum FRs can be immediately obtained and they resemble the classical ones. In this proposal the system does not interact with the environment and, thus, the dynamics is unitary.

To circumvent the problem of extending the TMP approach to an open system, ${ }^{17,18}$ we introduce work in analogy to that in the classical case as an integral of the injected power during the evolution. Let the evolution of the system be governed by a time-dependent Hamiltonian $\hat{H}(t)$ driven by a control parameter $\lambda(t)$. The corresponding power operator is then given by $\hat{P}=\partial \hat{H} / \partial \lambda \dot{\lambda}=\partial \hat{H} / \partial t$. If the state of the system is described by its reduced density operator $\hat{\rho}(t)$, the average power is given by $\langle\hat{P}(t)\rangle=\operatorname{Tr}\{\hat{\rho}(t) \hat{P}(t)\}$ and the expectation value of the work done on the quantum system is

$$
\langle W\rangle=\int_{0}^{\mathcal{T}}\langle\hat{P}(t)\rangle d t
$$

This way, the work explicitly depends on the whole evolution of the system through $\hat{\rho}(t)$ containing the information about the dynamics which can or cannot be unitary. To address this point, we differentiate the average energy of the system, $\langle\hat{H}\rangle=$
$\operatorname{Tr}\{\hat{\rho}(t) \hat{H}(t)\}$, yielding

$$
\frac{d}{d t}\langle\hat{H}\rangle=\operatorname{Tr}\left\{\frac{d \hat{\rho}}{d t} \hat{H}\right\}+\langle\hat{P}\rangle .
$$

Under quite general assumptions the dynamics of the reduced density operator of the system can be described by a master equation ${ }^{19} d \hat{\rho} / d t=-\frac{i}{\hbar}[\hat{H}, \hat{\rho}]+\hat{\mathcal{L}}(\rho)$, where the contributions on the right-hand side are given by the unitary and dissipative dynamics, respectively. By substituting the above result into Eq. (2), we find that there is no contribution due to the unitary dynamics since $\operatorname{Tr}\{[\hat{H}, \hat{\rho}] \hat{H}\}$ vanishes. Then the average power reads $\langle\hat{P}(t)\rangle=d\langle\hat{H}(t)\rangle / d t-\operatorname{Tr}\{\hat{\mathcal{L}}(\rho) \hat{H}(t)\}$ and the corresponding average work is given by

$$
\langle W\rangle=\langle\hat{H}(\mathcal{T})\rangle-\langle\hat{H}(0)\rangle-\int_{0}^{\mathcal{T}} d t \operatorname{Tr}\{\hat{\mathcal{L}}(\rho) \hat{H}(t)\} .
$$

If the system does not interact with the environment, only the first difference on the right-hand side survives in Eq. (3), and the average work is equal to the variation of the internal energy. The last term describes the energy exchange with the environment during the evolution process, and is dependent on the particular realization of the evolution trajectory. We call this contribution average heat and denote it as $Q$. In thermodynamical terms, Eq. (3) is the first law in the quantum regime, and it has been discussed previously in Refs. 20 and 21 as the energy balance equation.

The average work definition in Eq. (3) is more general than the TMP since it takes into account the full quantum evolution. For a closed quantum system, given the initial quantum state and a driving protocol, the evolution is determined completely by the Schrödinger equation, while the initial measurement performed in the TMP causes a collapse of the quantum state before the beginning of the protocol. Thus, the two approaches yield different results for the initial states with coherent superpositions of the eigenstates of $\hat{H}(0)$ and the TMP result is recovered if the system is initially in an eigenstate or in an incoherent superposition of the eigenstates of $\hat{H}(0)$. The second advantage of Eq. (3) is that it allows one to define and calculate the heat in terms of the system density matrix only, without detailed knowledge of the environment degrees of freedom. In addition, Eq. (3) can be used with any master equation. 


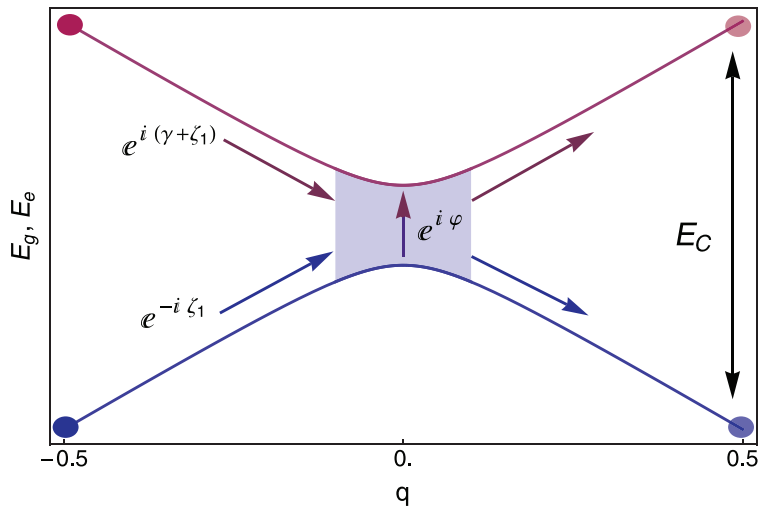

FIG. 1. (Color online) Schematic presentation of avoided crossing with the eigenstates of energies $E_{g}$ and $E_{e}$ in a CPB as a function of the normalized gate charge $q$. The phases which contribute to the interference are explicitly indicated.

Cooper-pair box as a driven quantum two-level system. We consider a Cooper-pair box $(\mathrm{CPB})^{22-24}$ consisting of a superconducting island connected to a superconducting lead by a Josephson tunnel junction. The system is described by the circuit scheme in the inset in Fig. 2 and it is characterized by a voltage source $V_{g}$, coupling gate capacitance $C_{g}$, a Josephson junction with energy $E_{J}$, and capacitance $C_{J}$. We denote $C_{\Sigma} \equiv C_{g}+C_{J}$. Resistor $R$, to be discussed in the last part of this Rapid Communication, forms the dissipative environment of the box. This system is a candidate for a calorimetric measurement of quantum work distribution. ${ }^{25}$

In the regime $\epsilon \equiv E_{J} /\left(2 E_{C}\right) \ll 1$, where $E_{C}=2 e^{2} / C_{\Sigma}$ is the charging energy of the box, we can treat the CPB as a two-level quantum system. Denoting with $|0\rangle$ and $|1\rangle$ the state with zero and one excess Cooper pair on the island, respectively, the Hamiltonian reads

$$
\hat{H}=-E_{C} q(|1\rangle\langle 1|-| 0\rangle\langle 0|)-\frac{E_{J}}{2}(|1\rangle\langle 0|+| 0\rangle\langle 1|),
$$

where $q=C_{g} V_{g} /(2 e)-1 / 2$ is the normalized gate voltage. We assume driven evolution: a linear gate $\operatorname{ramp} q(t)=$ $-1 / 2+t / \mathcal{T}$ over a period $\mathcal{T}$ starting from $t=0$. The ground and the excited states of the system are separated by the energy gap $\hbar \omega_{0}=2 E_{C} \sqrt{q^{2}+\epsilon^{2}}$ which reaches the minimum $E_{J}$ at $t=\mathcal{T} / 2$ (see Fig. 1). We recover the standard Landau-Zener (LZ) model, ${ }^{26,27}$ where the system is excited when driven in a nonadiabatic way through a avoided crossing.

The time-dependent eigenstates of the Hamiltonian (4) are $|g\rangle=\frac{1}{\sqrt{2}}(\sqrt{1-\eta}|0\rangle+\sqrt{1+\eta}|1\rangle)$ and $|e\rangle=$ $\frac{1}{\sqrt{2}}(\sqrt{1+\eta}|0\rangle-\sqrt{1-\eta}|1\rangle)$, where $\eta=q / \sqrt{q^{2}+\epsilon^{2}} \cdot{ }^{28}$

For this system, the power operator is $\hat{P}=E_{C} \dot{q}(\mathbb{1}-2 \hat{n})$, where $\hat{n}=|1\rangle\langle 1|$ is the operator of the number of Cooper pairs on the island and $\mathbb{1}=|1\rangle\langle 1|+| 0\rangle\langle 0|$ is the identity operator. We calculate the time-dependent average of the power operator in the Heisenberg picture, $\hat{P}^{H}(t)=U^{\dagger}(t) \hat{P} U(t)$, with the time evolution operator $U(t)$, and the state $|\psi(0)\rangle$ that does not change in time. Here we focus on the first and second moments of the work done on the CPB, which can be expressed through $\hat{P}^{H}(t)$ as $\langle W\rangle=\int_{0}^{\mathcal{T}} d t\left\langle\hat{P}^{H}(t)\right\rangle$ and $\left\langle W^{2}\right\rangle=\int_{0}^{\mathcal{T}} d t_{2} \int_{0}^{\mathcal{T}} d t_{1}\left\langle\hat{P}^{H}\left(t_{2}\right) \hat{P}^{H}\left(t_{1}\right)\right\rangle$, where $\langle\cdots\rangle \equiv$ $\langle\psi(0)|\cdots| \psi(0)\rangle$. Explicitly,

$$
\langle W\rangle=E_{C}\left(1-\frac{2}{\mathcal{T}} \int_{0}^{\mathcal{T}}\left\langle\hat{n}^{H}(t)\right\rangle d t\right)
$$

and

$$
\begin{aligned}
\left\langle W^{2}\right\rangle= & 2 E_{C}\langle W\rangle \\
& -E_{C}^{2}\left[1-\frac{4}{\mathcal{T}^{2}} \int_{0}^{\mathcal{T}} d t_{2} \int_{0}^{\mathcal{T}} d t_{1}\left\langle\hat{n}^{H}\left(t_{2}\right) \hat{n}^{H}\left(t_{1}\right)\right\rangle\right] .
\end{aligned}
$$

Equations (5) and (6) can be applied for both closed and open systems. ${ }^{19,29,30}$

Instantaneous transition regime, unitary evolution. If the time of the control ramp is much shorter than the relaxation and dephasing times, the evolution of the system can be considered unitary. For $\epsilon \ll 1$, the LZ transitions are localized near the minimum energy gap at $t=\mathcal{T} / 2$ and the dynamics is well approximated by the instantaneous transition model, ${ }^{31,32}$ i.e., the evolution is composed of pure adiabatic evolution and instantaneous LZ transitions at $t=\mathcal{T} / 2$ (see Fig. 1). All work, spent exactly in these LZ transitions, is stored in the system (CPB) as increased internal energy. Along the adiabatic region, the evolution operator reads $U_{k}(t)=\exp \left[-i \xi_{k}^{t} \sigma_{z}\right]$, where $\xi_{k}^{t}=\int_{t_{k-1}}^{t_{k}} d \tau \omega_{0}(\tau) / 2$ is half of the integrated energy gap, $t_{k}$ and $t_{k-1}$ denote the time limits of the adiabatic evolution, and $\sigma_{z}=$ $|e(t)\rangle\langle e(t)|-| g(t)\rangle\langle g(t)|$. The transfer matrix for the instantaneous LZ transitions in the basis $\{|g(\mathcal{T} / 2)\rangle,|e(\mathcal{T} / 2)\rangle\}$ reads

$$
N_{\mathrm{LZ}}=\left(\begin{array}{cc}
\sqrt{1-P_{\mathrm{LZ}}} e^{i \varphi} & -\sqrt{P_{\mathrm{LZ}}} \\
\sqrt{P_{\mathrm{LZ}}} & \sqrt{1-P_{\mathrm{LZ}}} e^{-i \varphi}
\end{array}\right),
$$

where $\varphi=\delta(\log \delta-1)+\arg \Gamma(1-i \delta)-\pi / 4$ is the gamma function). ${ }^{31,32}$ The probability of the LZ transition is given by $P_{\mathrm{LZ}}=e^{-2 \pi \delta}$, where $\delta=E_{J}^{2} \mathcal{T} /\left(8 E_{C}\right)=\epsilon^{2} E_{C} \mathcal{T} / 2$. We consider the system initially in a superposition of ground and excited state and $\left|\psi_{0}\right\rangle=\alpha|g(0)\rangle+\sqrt{1-\alpha^{2}} \exp (i \gamma)|e(0)\rangle$. We can then write for $t<\mathcal{T} / 2, U(t)=U_{1}(t)$, while after the LZ transition at $t>\mathcal{T} / 2, U(t)=U_{2}(t) N_{L Z} U_{1}(\mathcal{T} / 2)$. The calculation of $|\psi(t)\rangle$ is given in the Supplemental Material (SM). ${ }^{33}$ Here we discuss only the relevant results.

From Eq. (5), the corresponding average work is

$$
\begin{aligned}
\langle W\rangle= & E_{C}\left[\left(2 \alpha^{2}-1\right) P_{\mathrm{LZ}}+2 \alpha \sqrt{1-\alpha^{2}} \sqrt{\left(1-P_{\mathrm{LZ}}\right) P_{\mathrm{LZ}}}\right. \\
& \left.\times \cos \left(\gamma+\varphi+2 \xi_{1}^{\frac{\mathcal{\tau}}{2}}\right)\right]
\end{aligned}
$$

The first term represents the work done on the system which is initialized in the ground or in the excited state, i.e., $\alpha=$ 1 or 0 , respectively. The second term with its characteristic oscillatory behavior is due to the quantum interference at the LZ avoided crossing. ${ }^{32}$ This additional contribution is always present when the system is initially in a coherent state and it is a clear difference with the respect to the TMP. This difference is highlighted in Fig. 2(a), where we plot the analytical result in Eq. (8) as a function of $\mathcal{T}$ for different initial states. The oscillating behavior of $\langle W\rangle$ is obtained for $\alpha=1 / \sqrt{2}$ and $\gamma=0$ and we should compare it with the prediction of the TMP $\langle W\rangle=0$. The two exponential decays with $P_{\mathrm{LZ}}$ are obtained for the ground and excited initial states, $\alpha=1$ and $\alpha=0$ with 


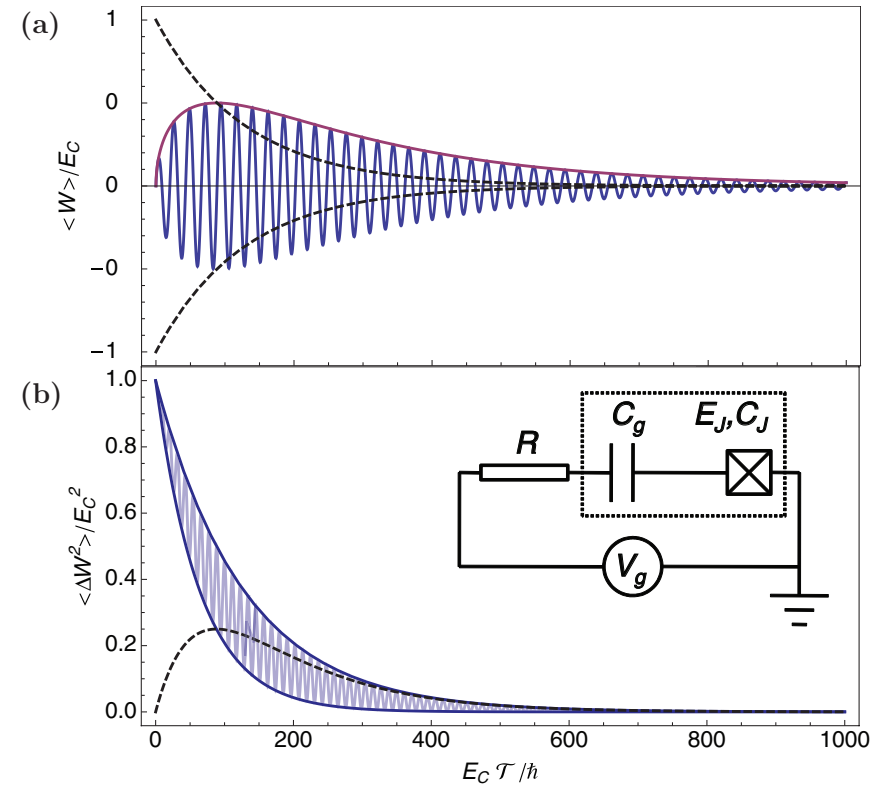

FIG. 2. (Color online) (a) Average work $\langle W\rangle$ normalized to $E_{C}$ for different initial states: $\alpha=1 / \sqrt{2}$ and $\gamma=0$ (blue oscillating curve), ground state $\alpha=1$ and $\gamma=0$ (top black exponentially decaying curve), and excited state $\alpha=0$ and $\gamma=0$ (lower black exponentially decaying curve). The purple curve denotes the behavior $\sqrt{P_{\mathrm{LZ}}\left(1-P_{\mathrm{LZ}}\right)}$. (b) Work variance $\left\langle\delta W^{2}\right\rangle$ normalized to $E_{C}^{2}$. The blue solid lines contain the oscillatory behavior for initial state $\alpha=1 / \sqrt{2}$ and $\gamma=0$. The black dashed line is obtained for the initial state, $\alpha=$ 1 and $\gamma=0$ (ground state). We have used $\epsilon=E_{J} /\left(2 E_{C}\right)=0.05$. Inset: Circuit scheme of the Cooper-pair box (CPB) connected to a dissipative environment $R$.

$\gamma=0$, respectively. The behavior for the thermalized initial density matrix can be obtained from these two curves with the correct weighted average.

With the same approach, the evaluation of Eq. (6) yields $\left\langle W^{2}\right\rangle=P_{\mathrm{LZ}} E_{C}^{2}$ for the second moment, independent of the initial state. With these results the corresponding rms fluctuation of work can be immediately calculated as $\left\langle\delta W^{2}\right\rangle=$ $\left\langle W^{2}\right\rangle-\langle W\rangle^{2}$. Figure 2(b) shows the behavior of $\left\langle\delta W^{2}\right\rangle$ for different initial states. Numerical simulations confirm the analytical results presented in Fig. 2.

The definition of work in Eq. (3) and the TMP give the same results if the system is initially in an eigenstate of $\hat{H}(0)$ or an incoherent superposition of them. In the interesting case in which the system is initialized in the ground state, i.e., $\alpha=1$, and for nearly adiabatic drive $\left(P_{\mathrm{LZ}} \ll 1\right)$, we have a linear response result linking the average work and its fluctuations as $\left\langle\delta W^{2}\right\rangle=E_{C}\langle W\rangle$.

In this specific case, the first two moments of work calculated above agree with the full work distribution $\rho(W)$ which for a closed system with unitary evolution $U(\mathcal{T})$ can be found essentially by direct comparison of the initial, $\hat{H}(0)$, and final, $\hat{H}(\mathcal{T})$, Hamiltonian of the system. Indeed, the work generating function $G(u)$ (Fourier transform of the distribution) can be written as (see, e.g., Ref. 11) $G(u)=$ $\operatorname{Tr}\left\{U^{\dagger}(\mathcal{T}) e^{i u \hat{H}(\mathcal{T})} U(\mathcal{T}) e^{-i u \hat{H}(0)} \rho_{0}\right\}$, where $\rho_{0}$ is the initial density matrix of the system assumed to be diagonal together with the initial Hamiltonian $\hat{H}(0)$. For the CPB considered above and the system initialized in the ground state, this equation gives $G(u)=1+P_{\mathrm{LZ}}\left(e^{i u E_{C}}-1\right)$, which corresponds to the following work distribution:

$$
\rho(W)=\left(1-P_{\mathrm{LZ}}\right) \delta(W)+P_{\mathrm{LZ}} \delta\left(W-E_{C}\right) .
$$

This distribution agrees with the first two moments and can be used to find the higher moments.

Open system with slow and fast relaxation. The most interesting and nontrivial test of Eq. (3) is when the system interacts with the environment during the evolution of $q$. To evaluate the heat contribution in Eq. (3) we need to consider a concrete example of the system-environment interaction. If the time and the ramp time are of the same order, dissipation $Q$ takes place during the driven evolution. To evaluate dissipation during the sweep, we then solve the master equation (ME) of the CPB adapted from the corresponding ME of Refs. 28 and 34. This ME and some details of the analysis are given in the SM. ${ }^{33}$ The environment is described by the resistor $R$ coupled capacitively to the island of the CPB (inset in Fig. 2). As above, we assume that the temperature is low as compared to the excitation energy. If the system is initially in the ground state, the average heat released to the environment during the ramp normalized by the total work done for a few values of $\epsilon$ is shown in Fig. 3(a) based on the numerical solution of the
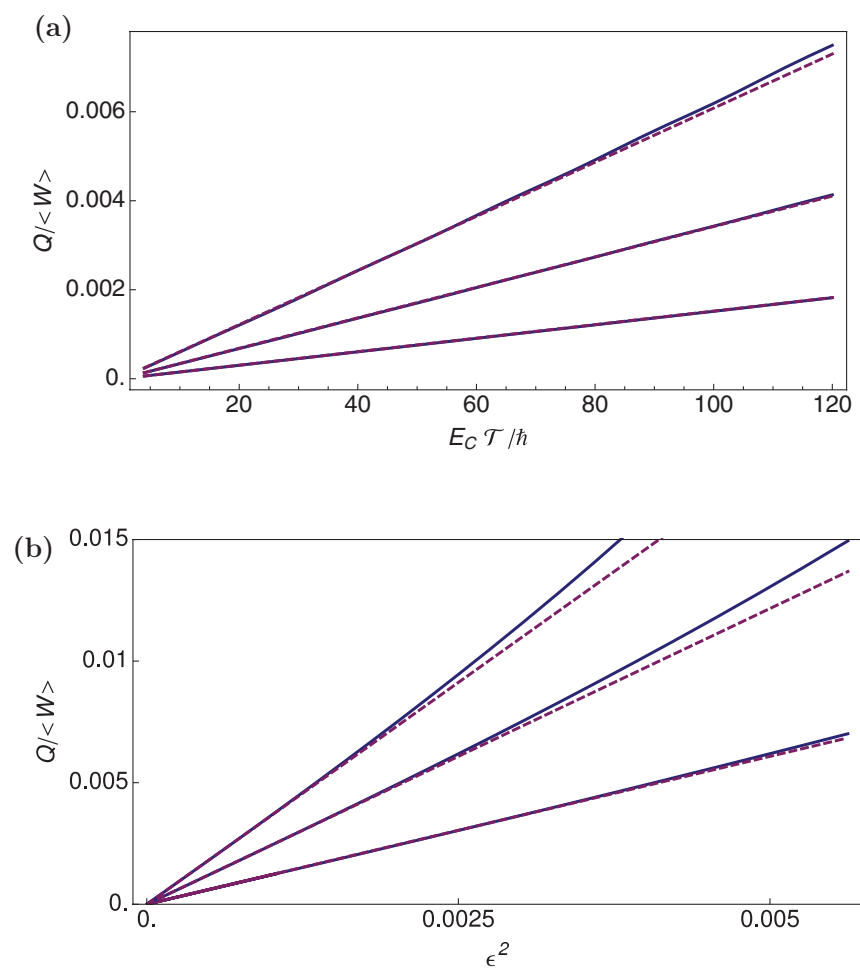

FIG. 3. (Color online) (a) Numerically calculated (solid lines) dissipated average heat during the sweep in an open CPB as a function of the sweep time when the system is initially in the ground state. The system-environment coupling constant is chosen to be $C_{g} / C_{\Sigma}=$ 0.05 here, $E_{C} / k_{B}=1 \mathrm{~K}$, and the environment resistance is $R=$ $1 \times 10^{4} \Omega$. The different curves from top to bottom correspond to $\epsilon=0.05,0.0375$, and 0.025 . The dashed line is the analytic approximation of Eq. (10). (b) Dissipated average heat at $E_{C} \mathcal{T} / \hbar=150,100$, and 50 from top to bottom as a function of $\epsilon^{2}$. The other parameters and the line conventions are as in (a). 
ME (solid lines). The dependence of the same quantity on the minimum energy gap $E_{J}$ (for scaling purposes the horizontal axis is $\epsilon^{2}$ ) is shown in Fig. 3(b). The apparent dependences on the various parameters in Fig. 3 are captured by a simple analytical approximation

$$
Q /\langle W\rangle \simeq \frac{R}{R_{Q}}\left(\frac{C_{g}}{C_{\Sigma}}\right)^{2}\left(\frac{E_{J}}{E_{C}}\right)^{2} \frac{E_{C} \mathcal{T}}{\hbar},
$$

which is derived in the SM (Ref. 33) with the assumption that, again, the LZ transition occurs exactly at $t=\mathcal{T} / 2$ with probability $P_{\mathrm{LZ}}$. Here, $R_{Q} \equiv \hbar / e^{2}$. The prediction of Eq. (10) is shown by dashed lines in Fig. 3, in close agreement with the full numerical solution. Energy relaxation occurs uniformly over the positive values of $q$, leading to the proportionality of $Q /\langle W\rangle$ on $\mathcal{T}$. As a by-product, Fig. 3(a) justifies the semiquantitative analysis and the proposed measurement protocol above, since most of the work remains stored in the system during fast ramps (small $\mathcal{T}$ ).

In the limit of fast relaxation, i.e., $\tau \ll \mathcal{T}$, it is possible to obtain an analytical estimate of the heat released during the evolution. Since the dephasing time is usually smaller than the relaxation time, we can assume that the coherences between the ground and excited state are quickly lost and they do not influence the dynamics. The system tends to follow the instantaneous equilibrium state but due to the drive there are corrections to this dynamics which cause the heat emission. We calculate the heat in this regime directly from the semiclassical master equation. As shown in $\mathrm{SM},{ }^{33}$ the same result is still obtained if one takes into account quantum corrections from the finite drive to adiabatic dynamics.

The integrand in the heat contribution in Eq. (3) can be conveniently written as $-\hbar \dot{\rho}_{g g} \omega_{0}$. If we denote with $\Gamma_{g e}$ and $\Gamma_{e g}$ the excitation and relaxation rates, respectively, in the semiclassical limit we have $\dot{\rho}_{g g}=-\Gamma_{\Sigma} \rho_{g g}+\Gamma_{g e}$, where $\Gamma_{\Sigma}=\Gamma_{g e}+\Gamma_{e g}, \Gamma_{g e} / \Gamma_{e g}=\exp \left(-\beta \hbar \omega_{0}\right)$, and $\beta$ is the inverse temperature of the environment. We use the trial solutions $\rho_{g g}=\rho_{g g}^{(0)}+\delta \rho_{g g}^{(1)}$, where $\rho_{g g}^{(0)}=\Gamma_{g e} / \Gamma_{\Sigma}$ is the stationary solution and $\delta \rho_{g g}^{(1)}$ is the correction due to the drive. Plugging $\rho_{g g}$ in the initial equation we obtain $\delta \rho_{g g}^{(1)}=$ $-\dot{\rho}_{g g}^{(0)} / \Gamma_{\Sigma}$. When integrated the adiabatic contribution $\hbar \dot{\rho}_{g g}^{(0)} \omega_{0}$ gives no contribution (see $\mathrm{SM}^{33}$ ) and, with an integration by parts, the nonvanishing contribution in the limit $\beta E_{C} \gg 1$ can be written as

$$
\begin{aligned}
Q & =\frac{4 E_{C}^{2}}{\hbar \mathcal{T}} \int_{-\frac{1}{2}}^{\frac{1}{2}} d q \frac{q}{\omega_{0} \Gamma_{\Sigma}} \frac{d}{d q}\left(\frac{\Gamma_{e g}}{\Gamma_{\Sigma}}\right) \\
& =\frac{\beta E_{C}^{2}}{\mathcal{T}} \int_{-\frac{1}{2}}^{\frac{1}{2}} d q \frac{\eta^{2}}{\Gamma_{\Sigma} \cosh ^{2}\left(\frac{\beta \omega_{0}}{2}\right)} .
\end{aligned}
$$

Here the second line arises from the detailed balance of the transition rates. Thus, we recover the expected properties of the released heat: (i) It depends on the full evolution which, in this limit, is represented by the driving parameter $q$, (ii) it scales as $1 / \mathcal{T}$, and (iii) it is positive. ${ }^{35}$

In summary, we have analyzed work done by a driving field on a quantum system. The obtained expression of average work has a physical interpretation, allowing one to assign separate contributions to the change in the internal energy and the heat dissipated to the environment in the spirit of the first law of thermodynamics. We applied our results to a two-level system, obeying in the first case unitary evolution and then in the presence of weak dissipation. For an open system, we presented a detailed analysis of the released heat two regimes where the relaxation time was either of the order or smaller than the driving time. In the latter case, our approach allows an analytical calculation of the released heat which has and immediate physical interpretation.

We would like to thank T. Ala-Nissilä, A. Kutvonen, S. Suomela, S. Gasparinetti, M. Möttönen, and J. Ankerhold for useful discussions. This work was supported by the European Community FP7 under Grant No. 238345 GEOMDISS and Academy of Finland Centre of Excellence. P.S. acknowledges financial support from FIRB Đ Futuro in Ricerca 2012 under Grant No. RBFR1236VV HybridNanoDev.
${ }^{1}$ G. N. Bochkov and Yu. E. Kuzovlev, Sov. Phys. JETP 45, 125 (1977).

${ }^{2}$ C. Jarzynski, Phys. Rev. Lett. 78, 2690 (1997).

${ }^{3}$ A. Alemany, M. Ribezzi, and F. Ritort, in Proceedings of the 11th Granada Seminar on Computational and Statistical Physics, edited by P. L. Garrido, J. Marro, and F. de los Santos, AIP Conf. Proc. Vol. 1332 (AIP, Melville, NY, 2011), p. 96110.

${ }^{4}$ D. V. Averin and J. P. Pekola, Europhys. Lett. 96, 67004 (2011).

${ }^{5}$ B. Küng et al., Phys. Rev. X 2, 011001 (2012).

${ }^{6}$ O. P. Saira, Y. Yoon, T. Tanttu, M. Mottonen, D. V. Averin, and J. P.

Pekola, Phys. Rev. Lett. 109, 180601 (2012).

${ }^{7}$ S. Yukawa, J. Phys. Soc. Jpn. 69, 2367 (2000).

${ }^{8}$ V. Chernyak and S. Mukamel, Phys. Rev. Lett. 93, 048302 (2004).

${ }^{9}$ A. E. Allahverdyan and Th. M. Nieuwenhuizen, Phys. Rev. E 71, 066102 (2005).

${ }^{10}$ A. Engel and R. Nolte, Europhys. Lett. 79, 10003 (2007).
${ }^{11}$ P. Talkner, E. Lutz, and P. Hänggi, Phys. Rev. E 75, 050102(R) (2007).

${ }^{12}$ M. Campisi, P. Hänggi, and P. Talkner, Rev. Mod. Phys. 83, 711 (2011); 83, 1653(E) (2011).

${ }^{13}$ J. Kurchan, arXiv:cond-mat/0007360.

${ }^{14} \mathrm{H}$. Tasaki, arXiv:cond-mat/0009244.

${ }^{15}$ S. Mukamel, Phys. Rev. Lett. 90, 170604 (2003).

${ }^{16}$ M. Esposito, U. Harbola, and S. Mukamel, Rev. Mod. Phys. 81, 1665 (2009).

${ }^{17}$ M. Campisi, P. Talkner, and P. Hänggi, Phys. Rev. Lett. 102, 210401 (2009).

${ }^{18}$ P. Talkner, M. Campisi, and P. Hänggi, J. Stat. Mech. (2009) P02025.

${ }^{19}$ H. P. Breuer and F. Petruccione, The Theory of Open Quantum Systems (Oxford University Press, Oxford, 2002).

${ }^{20}$ A. E. Allahverdyan and Th. M. Nieuwenhuizen, Phys. Rev. E 64, 056117 (2001). 
${ }^{21}$ M. Esposito and S. Mukamel, Phys. Rev. E 73, 046129 (2006).

${ }^{22}$ D. V. Averin, A. B. Zorin, and K. K. Likharev, Sov. Phys. JETP 88, 692 (1985).

${ }^{23}$ V. Bouchiat, D. Vion, P. Joyez, D. Esteve, and M. H. Devoret, Phys. Scr., T 76, 165 (1998).

${ }^{24}$ Y. Nakamura, Yu. A. Pashkin, and J. S. Tsai, Nature (London) 398, 786 (1999).

${ }^{25}$ J. P. Pekola, P. Solinas, A. Shnirman, and D. V. Averin, arXiv: 1212.5808 .

${ }^{26}$ C. Zener, Proc. R. Soc. London, Ser. A 137, 696 (1932).

${ }^{27}$ M. Campisi, P. Talkner, and P. Hänggi, Phys. Rev. E 83, 041114 (2011).

${ }^{28}$ P. Solinas, M. Möttönen, J. Salmilehto, and J. P. Pekola, Phys. Rev. B 82, 134517 (2010).
${ }^{29}$ Quantum Noise in Mesoscopic Physics, edited by Yu. V. Nazarov (Kluwer, Dordrecht, The Netherlands, 2003).

${ }^{30} \mathrm{C}$. Gardiner and P. Zoller, Quantum Noise, 3rd ed. (Springer, Berlin, 2004).

${ }^{31}$ S. Shevchenko, S. Ashhab, and F. Nori, Phys. Rep. 492, 1 (2010).

${ }^{32}$ S. Gasparinetti, P. Solinas, and J. P. Pekola, Phys. Rev. Lett. 107, 207002 (2011)

${ }^{33}$ See Supplemental Material at http://link.aps.org/supplemental/ 10.1103/PhysRevB.87.060508 for more technical details.

${ }^{34}$ J. P. Pekola, V. Brosco, M. Möttönen, P. Solinas, and A. Shnirman, Phys. Rev. Lett. 105, 030401 (2010).

${ }^{35} \mathrm{~K}$. Sekimoto, Stochastic Energetics, Lecture Notes in Physics Vol. 799 (Springer, Berlin, Heidelberg, 2010). 